\title{
La influencia de la gestión del cambio en la implementación de BIM en la industria de la construcción sostenible de México
}

\author{
Yaniel Llerena Padilla a, ${ }^{\text {a, }}$, Carlos Alfredo Bigurra-Alzatib \\ a Programa de Maestría y Doctorado en Arquitectura de la Universidad Nacional Autónoma de México. \\ ${ }^{b}$ Área Académica de Ingeniería y Arquitectura, UAEH, Mineral de la Reforma, Hidalgo.
}

\begin{abstract}
Resumen
BIM ha puesto en crisis el modo de producción tradicional de la industria para centrarse en los procesos colaborativos y de gestión de información como pilares fundamentales de un nuevo paradigma. A pesar de sus reconocidas bondades, la implementación y difusión de BIM se encuentran con numerosas barreras que, a pesar de incluir la dimensión tecnológica, trascienden a la dimensión social. Esta última se expresa en el cambio de metodologías de trabajo, en nuevas formas de colaboración, en el diseño y construcción sostenible, en la aparición de nuevos roles, en los ajustes de organigramas empresariales, de contratos y de modelos de negocio. Todos estos aspectos tienen además un factor común que es el cambio, elemento donde el proceso de implementación de BIM encuentra su mayor obstáculo debido a la innovación que representa para cualquier organización. Con la presente investigación se lleva a cabo una correlación estadística para determinar la influencia de la Gestión del Cambio en el proceso de implementación de BIM. Se pretende con ello ofrecer mayor claridad en el posible uso de la Gestión del Cambio para conducir un proceso disruptivo y con altos riesgos asociados como lo es la implementación BIM en una organización u empresa. Se procura también crear conciencia sobre la importancia e impacto de la dimensión social de BIM en cualquier proyecto de implementación que busque ser realmente exitoso.
\end{abstract}

Palabras Clave: BIM, CM, Modelado de Información para la Construcción, Gestión del Cambio.

\section{Introducción}

El concepto de BIM (Building Information Modeling) ha puesto en crisis el modo de producción tradicional de la industria AECO (por sus siglas en inglés Architecture, Engeneering, Construction and Operations Industry) para centrarse en los procesos colaborativos y de gestión de información como pilares fundamentales de un nuevo paradigma. Para cumplir con este cometido, BIM ha estado asociado al amplio desarrollo de las tecnologías de la información. Precisamente este hecho ha incidido en que, de forma reiterada tanto el ámbito científico como profesional, trabajen BIM solo desde su arista tecnológica. Cabe destacar que, a pesar de sus reconocidas bondades, la implementación y difusión de BIM se encuentran con numerosas barreras que, a pesar de incluir la dimensión tecnológica, trascienden a la dimensión social. Esta última se expresa en el cambio de metodologías de trabajo e incluir el diseño y construcción sostenible, en nuevas formas de colaboración, en la aparición de nuevos roles, en los ajustes de organigramas empresariales, de contratos y de modelos de negocio. Todos estos aspectos tienen además un factor común que es el cambio, elemento donde el proceso de implementación de BIM encuentra su mayor obstáculo debido a la innovación que representa para cualquier organización.

Con la presente investigación se aborda BIM desde una perspectiva socio-técnica con el fin de arrojar luz sobre sus retos en la dimensión social y la oportunidad de utilizar los

* Autor en correspondencia.

Correos electrónicos: yllerena1987@gmail.com (Yaniel Llerena Padilla) conocimientos de otras disciplinas como la Gestión del Cambio. Para ello nos planteamos como objetivo determinar el grado de correlación que existe entre el Nivel de Madurez BIM y el Nivel de Madurez de la Gestión del Cambio alcanzados por las empresas mexicanas que tengan implementada dicha metodología. Se realiza además una caracterización de BIM y de la Gestión del Cambio desde el punto de vista teórico; se identifican los modelos existentes de Madurez de BIM y de la Gestión del Cambio y se evalúan herramientas existentes para medir ambos términos en empresas mexicanas que utilizan BIM en el día a día. Finalmente se lleva a cabo una correlación estadística para determinar, describir y explicar la influencia de la Gestión del Cambio en el proceso de implementación de BIM. Con todo ello, se pretende ofrecer mayor claridad en el posible uso de la Gestión del Cambio para conducir un proceso disruptivo y con altos riesgos asociados como lo es la implementación BIM en una organización u empresa. También se pretende crear conciencia sobre la importancia e impacto de la dimensión social de BIM en cualquier proyecto de implementación que busque ser realmente exitoso.

\section{Materiales y métodos}

\subsection{Building Informatión Modeling (BIM)}

El concepto de BIM ha sido objetivo de disímiles interpretaciones, situación que puede acarrear confusiones en 
los profanos interesados. A pesar de este hecho, algunos de los esfuerzos realizados por parte de investigadores y practicantes, van enfocados hacia una definición de BIM integradora, acorde con su desarrollo teórico- práctico que cada día es más abarcador (Eastman et al., 2011). Un concepto que se mantiene firme dentro de las definiciones encontradas, es que BIM es tanto una herramienta como un proceso. Para los propósitos de esta investigación, se han tomado como base las definiciones de BIM afines a la visión integradora antes mencionada.

En BIM Handbook: A Guide to Building Information Modeling for Owners, Managers, Designers, Engineers and Contractors, el autor (Chuck) hacen referencia a la definición BIM aportada por la National Building Information Modeling Standard (NBIMS):

'BIM es un proceso mejorado de planeación, diseño, construcción, operación y mantenimiento, basado en un modelo de información estandarizado y legible para cada obra, nueva o vieja, el cual contiene toda la información apropiada, creada u obtenida, sobre dicha obra en un formato usable durante todo su ciclo de vida.'

En una aproximación similar en su libro BIM and Construction Management: Proven tools, methods, and workflows, plantean que: 'el uso exitoso de BIM requiere de tres factores: procesos, tecnologías y comportamientos.' El factor comportamientos, se señala como uno de los más importantes debido a que es el más difícil de modificar:

'Cuando nos planteamos qué hace que BIM funcione, el componente principal se traduce en inducir comportamientos. Los equipos deben comprender que mantener un estado mental enfocado al futuro, es tan importante como la tecnología y los procesos detrás de ella...es importante también notar cómo el comportamiento organizacional puede impactar en la exitosa integración de la tecnología. Una compañía que tenga una cultura de innovación...creará una dinámica persistente donde el cambio será una constante, así como la mejora y el análisis.'

Una definición que resume las anteriormente citadas, en una serie de políticas, procesos y tecnologías interactivas que generan una metodología para administrar los datos y el diseño de un proyecto en formato digital a través de su ciclo de vida.'

\subsection{La implementación y la adopción de BIM}

La implementación y la adopción de BIM son términos que suelen ser usados de forma indistinta. Este hecho tiende a crear cierta confusión entre los usuarios y las organizaciones interesadas en el tema. A menudo los usuarios usan ambos conceptos para referirse a la inversión y acción correspondiente de adquirir nuevas licencias e instalar nuevos programas.

Para fines de esta investigación se tomaron las definiciones establecidas en el modelo conceptual Point Of Adoption, el cual será referenciado a partir de este momento como PoA. Este modelo abarca e integra, de forma más profunda, los procesos de adopción e implementación, a la vez que introduce a la fórmula el término de difusión. Partiendo de sus criterios, la Implementación BIM será entendida como el conjunto de actividades llevadas a cabo por una unidad organizacional para preparar el despliegue o la optimización de los flujos de trabajos BIM y sus entregables (servicios o productos). En el modelo de PoA la implementación BIM se divide en 3 fases que definen la disposición de adoptar, la capacidad de ejecutar dicha disposición y la madurez alcanzada según su rendimiento a través del tiempo.

\subsection{La gestión del cambio}

El cambio es una condición inherente a la sociedad moderna y su importancia se acelera debido la escasez del recurso tiempo, la escasez del recurso dinero y el aumento de la complejidad. Por otra parte las crecientes presiones a las que están sometidas las organizaciones dentro del panorama económico actual han traído consigo que las empresas se enfrenten a procesos de adaptación constante. Del éxito y la culminación de dichos procesos depende en gran medida la supervivencia de las propias organizaciones. Si tomamos como una caracterización BIM aportada por Succar \& Kassem (2016), se puede entretejer la relación existente entre su significado y las implicaciones que tiene como cambio para una empresa u organización:

'BIM (...) necesitará ser reclasificada de forma urgente en base a su adopción transformadora- como una innovación organizacional caracterizada por la generación, la aceptación y la implementación de nuevas ideas, procesos, productos o servicios'

Sin duda la empresa que decida adoptar BIM se encontrará inmersa en un proceso de cambio. Para enfrentarlo, el primer paso será reconocerlo y admitirlo, el segundo digerirlo y conducirlo para que se convierta luego un verdadero progreso.

Según su origen, los cambios pueden clasificarse en dos categorías: el cambio no planeado, donde el empuje de las presiones externas hace del cambio un proceso inevitable, y el cambio planeado, el cual puede ser concebido y administrado. Por otra parte, la Gestión del Cambio ha sido abordada desde dos vertientes principales, la organizacional y la individual. La primera de ellas destaca en la sucesión de modelos desarrollados como los expuestos por:

- Modelo de los tres pasos

- Modelo del cambio planeado

- Modelo del cambio de sistema total

- Modelo del desempeño y cambio organizacional Modelo del análisis de flujo

- Modelo para la administración del cambio

- Modelo de los 8 pasos de Kotter

- Modelo del crecimiento

Metodología para la gestión del cambio organizacional

La mayoría de los modelos transitan por cuatro etapas generales (Lopez et al., 2013): determinar los objetivos del cambio de forma precisa y de la forma más clara posible. Crear una estrategia de innovación y cambio -"top down" o "bottom up"-. Diseñar el cambio organizacional de la empresa (impulsos, feedback, agente del cambio, jour fixes, workshop, perfeccionamiento, multiplicadores, Intranet, hojas informativas...). Mantener y consolidar el proceso de innovación (resistencias, crisis, superar fases difíciles).

Más allá de los numerosos modelos de cambio organizacional existentes, hemos constado que el cambio a nivel individual ha sido menos explorado. Sin embargo, autores como John Kotter en una publicación titulada The Heart of Change, 2012 presenta un nuevo enfoque de su modelo de los 8 pasos donde enfatiza que 'el problema 
fundamental en todas las etapas es cambiar el comportamiento de las personas'.

Uno de los modelos de cambio individual más reconocidos en la actualidad es el Modelo ADKAR. El mismo se basa en la premisa de que "para que un cambio en una organización sea exitoso, es necesario centrarse en cómo facilitar el cambio en una sola persona'.

ADKAR es un acrónimo que representa 5 aspectos claves que han de ser cumplidos o gestionados para que el cambio individual exista:
A: Awareness (Entendimiento)
D: Desire (Deseo)
$\mathrm{K}$ : Knowledge (Conocimiento)
A: Ability (Habilidad)
R: Reinforcement (Refuerzo)

\subsection{Los modelos de madurez de BIM y la gestión del cambio}

Los Modelos de Madurez son considerados como herramientas estratégicas que permiten dar seguimiento, priorizar y evaluar las capacidades de una organización en base a sus procesos y entregables. De forma general pueden ser descritos como 'las capacidades de una organización que evolucionan de un estado a otro a través de un camino lógico, deseado y anticipado'.

Para BIM han sido desarrollados diversos Modelos de Madurez y muchos de ellos vienen asociados a herramientas de medición complementarias. Varias investigaciones han realizado un análisis exhaustivo de los mismos donde podemos identificar los siguientes:

- National BIM Standard Capability Maturity Model (NBIMS-CMM) IU BIM Proficiency Index

- BIM Maturity Matrix BIM Quick Scan VDC Scorecard Owner's BIMCAT

- $\quad$ BIM Cloud Score (BIMCS)

- $\quad$ ARUPS's BIM Maturity Measure (BIM-MM)

De los Modelos de Madurez estudiados y sus herramientas podemos concluir que: el método de medición más utilizado son las escalas ordinales, la suma ponderada es el método de preferencia para el cálculo de las puntuaciones, y la herramienta de medición más utilizada es el cuestionario.

Desde el punto de vista de la Gestión del Cambio no hemos podido encontrar Modelos de Madurez específicos para la disciplina, más allá de lo propuesto por Procsi con su Modelo ADKAR. El mismo cuenta con una herramienta de medición que presenta las mismas características antes mencionadas de los Modelos de Madurez BIM.

\subsection{Población y tamaño de la muestra}

Nuestra población de estudio está compuesta por aquellas empresas que pertenecen a la Industria de la Construcción, con sede en la Ciudad de México y que tengan implementado BIM dentro de sus procesos productivos. Para delimitar la misma con mayor certeza se analizaron tres bases de datos:

Bases de datos comerciales

Base de datos de la SIEM

Base de datos DENUE del INEGI

Esta última fue la seleccionada para la investigación debido a su mayor certeza y mayor número de registros en comparación con las bases de datos comerciales y de la SIEM. Una vez revisada la base de datos se le aplicaron restricciones más allá de la actividad económica y la localización. De forma específica se filtraron aquellas empresas que presentan información de contacto vía email, pues debido al gran número de empresas, la vía más expedita para llegar a ellas y obtener datos es mediante el correo electrónico. Una vez aplicada la restricción el número de empresas fue de 402. Para determinar la población final de la presente investigación es necesario conocer cuántas empresas utilizan BIM. Este es un dato con el cual no se cuenta en la actualidad, por lo tanto será obtenido mediante una encuesta.

\subsection{Variables dependientes}

Las variables dependientes serán tomadas de la herramienta de ARUP que mide el Nivel de Madurez BIM y que fue introducida y nombrada anteriormente. En este sentido se medirán 22 variables descritas, pero para realizar las correlaciones serán agrupadas en tres variables principales:

Y1: Nivel de Madurez BIM Organizacional

Y2: Nivel de Madurez BIM Técnico

Y3: Nivel de Madurez BIM

Y1 y Y2 serán obtenidas a partir del promedio ponderado de las variables que las componen. Y3 será el promedio entre Y1 y Y2. Las variables son ordinales y serán medidas a través de una escala de Likert del 0 a 5 en total correspondencia con la herramienta de referencia:

$$
\begin{array}{ll}
0 & - \text { No existente } \\
1 & - \text { Inicial } \\
2 & - \text { Gestionado } \\
3 & - \text { Definido } \\
4 & - \text { Medido } \\
5 & - \text { Optimizado }
\end{array}
$$

\begin{tabular}{|c|c|c|}
\hline \multicolumn{3}{|c|}{ Variables del nivel de madurez BIM } \\
\hline & $\begin{array}{l}\text { Variable Y1: nivel de } \\
\text { madurez BIM } \\
\text { organizacional }\end{array}$ & Descripción \\
\hline $\begin{array}{c}\mathrm{Y} 1 \\
1\end{array}$ & $\begin{array}{l}\text { Requerimientos de } \\
\text { Información del } \\
\text { Cliente (EIR) }\end{array}$ & $\begin{array}{c}\text { Mide el nivel en que los requerimientos } \\
\text { del cliente han sido definidos e } \\
\text { implementados }\end{array}$ \\
\hline $\begin{array}{c}\mathrm{Y} 1 \\
2\end{array}$ & Revisión de datos BIM & $\begin{array}{l}\text { Mide el nivel en que se realizan } \\
\text { revisiones pre-licitación y post- } \\
\text { adjudicación para asegurar que los } \\
\text { requerimientos del clientes son } \\
\text { cumplidos }\end{array}$ \\
\hline $\begin{array}{c}\mathrm{Y} 1 \\
3\end{array}$ & $\begin{array}{l}\text { Plan de Ejecución } \\
\text { BIM (BEP) }\end{array}$ & $\begin{array}{c}\text { Mide el nivel en que se usa un Plan de } \\
\text { Ejecución BIM para formalizar cómo la } \\
\text { información será administrada y entrega } \\
\text { en relación a los requerimientos del } \\
\text { cliente }\end{array}$ \\
\hline $\begin{array}{l}\text { Y1 } \\
4\end{array}$ & $\begin{array}{l}\text { Obtención del } \\
\text { Proyecto }\end{array}$ & $\begin{array}{c}\text { Mide el nivel en que se considera a BIM } \\
\text { dentro de las discusiones con los } \\
\text { Contratistas }\end{array}$ \\
\hline $\begin{array}{c}\mathrm{Y} 1 \\
5\end{array}$ & $\begin{array}{l}\text { Entorno Común de } \\
\text { Datos (CDE) }\end{array}$ & $\begin{array}{l}\text { Mide el nivel en que se utiliza un CDE } \\
\text { para facilitar la información compartida }\end{array}$ \\
\hline $\begin{array}{c}\mathrm{Y} 1 \\
6\end{array}$ & $\begin{array}{l}\text { Versionado de } \\
\text { archivos, estatus }\end{array}$ & $\begin{array}{l}\text { Mide el nivel en que se llevan a cabo } \\
\text { procedimientos y métodos estandarizados } \\
\text { en la producción de los modelos }\end{array}$ \\
\hline $\begin{array}{c}\text { Y1 } \\
7\end{array}$ & $\begin{array}{l}\text { Estrategia de } \\
\text { Marketing }\end{array}$ & $\begin{array}{c}\text { Mide el nivel en que se preparan estudios } \\
\text { de caso específicos sobre BIM para } \\
\text { labores de marketing }\end{array}$ \\
\hline
\end{tabular}

Tabla 1: Variables de la herramienta BIM-MM (variables dependientes de la investigación). 


\begin{tabular}{|c|c|c|}
\hline $\begin{array}{c}\mathrm{Y} 1 \\
8\end{array}$ & $\begin{array}{l}\text { Revisiones de Diseño } \\
\text { Virtual }\end{array}$ & $\begin{array}{c}\text { Mide el nivel en que se realizan } \\
\text { revisiones virtuales antes de emitir los } \\
\text { modelos hacia las tareas de coordinación } \\
\text { y control de calidad } \\
\end{array}$ \\
\hline $\begin{array}{c}\text { Y1 } \\
9 \\
\end{array}$ & $\begin{array}{l}\text { Estándares Abiertos } \\
\text { BIM }\end{array}$ & $\begin{array}{l}\text { Mide el nivel en que los entregables son } \\
\text { verificados con especificaciones de } \\
\text { estándares abiertos como IFC y COBie }\end{array}$ \\
\hline $\begin{array}{c}\mathrm{Y} 1 \\
10\end{array}$ & Contratos BIM & $\begin{array}{c}\text { Mide el nivel en que el equipo de } \\
\text { proyecto está de acuerdo y firma } \\
\text { obligaciones contractuales con respecto a } \\
\text { BIM }\end{array}$ \\
\hline $\begin{array}{c}\mathrm{Y} 1 \\
11\end{array}$ & Campeón BIM & $\begin{array}{c}\text { Mide el nivel de madurez del equipo de } \\
\text { gestión del cambio (Campeón BIM y } \\
\text { equipo de Implementación BIM) en } \\
\text { apoyo a la adopción de BIM en la } \\
\text { organización }\end{array}$ \\
\hline \multicolumn{2}{|r|}{$\begin{array}{l}\text { Variable Y2: nivel de } \\
\text { madurez bim técnico }\end{array}$} & Descripción \\
\hline $\begin{array}{c}\mathrm{Y} 2 \\
1\end{array}$ & Modelo 3D & $\begin{array}{l}\text { Mide el nivel en que el modelo es usado } \\
\text { como parte de la coordinación de diseño } \\
\text { en las fases de diseño y construcción }\end{array}$ \\
\hline $\begin{array}{c}\mathrm{Y} 2 \\
2 \\
\end{array}$ & Dibujos & $\begin{array}{c}\text { Mide el nivel en que la documentación, } \\
\text { incluyendo los dibujos, son obtenidos } \\
\text { directamente de los modelos }\end{array}$ \\
\hline $\begin{array}{c}\mathrm{Y} 2 \\
3\end{array}$ & Niveles de Desarrollo & $\begin{array}{c}\text { Mide el nivel en que las salidas de diseño } \\
\text { son controladas para solo entregar la } \\
\text { información relevante en base a un nivel } \\
\text { de detalle definido para cada etapa del } \\
\text { proyecto }\end{array}$ \\
\hline $\begin{array}{c}\mathrm{Y} 2 \\
4\end{array}$ & $\begin{array}{l}\text { Revisiones y } \\
\text { coordinación }\end{array}$ & $\begin{array}{l}\text { Mide el nivel en que se aplican revisiones } \\
\text { y chequeos apropiados como parte del } \\
\text { proceso de intercambio de información }\end{array}$ \\
\hline $\begin{array}{c}\mathrm{Y} 2 \\
5\end{array}$ & $\begin{array}{l}\text { Inclusión de datos e } \\
\text { información }\end{array}$ & $\begin{array}{l}\text { Mide el nivel en que se añaden datos } \\
\text { relevantes al modelo, para su posterior } \\
\text { extracción y uso en todos los aspectos de } \\
\text { diseño y documentación }\end{array}$ \\
\hline $\begin{array}{c}\mathrm{Y} 2 \\
6 \\
\end{array}$ & Visualización & $\begin{array}{c}\text { Mide el nivel en que se utiliza la } \\
\text { visualización del modelo como una } \\
\text { herramienta de comunicación del diseño }\end{array}$ \\
\hline $\begin{array}{c}\text { Y2 } \\
7 \\
\end{array}$ & 4D - Tiempo & $\begin{array}{l}\text { Mide el nivel en que el modelo es usado } \\
\text { para facilitar la secuencia de construcción }\end{array}$ \\
\hline $\begin{array}{c}\mathrm{Y} 2 \\
7 \\
\end{array}$ & 5D - Costos & $\begin{array}{l}\text { Mide el nivel en que el modelo es usado } \\
\text { para extraer información de costo y } \\
\text { cantidades }\end{array}$ \\
\hline $\begin{array}{c}\mathrm{Y} 2 \\
8\end{array}$ & $\begin{array}{l}\text { 6D - Simulación y } \\
\text { Análisis }\end{array}$ & $\begin{array}{l}\text { Mide el nivel en que el modelo es usado } \\
\text { como parte de los procesos de análisis de } \\
\text { diseño y simulación (clima, energía, etc.) }\end{array}$ \\
\hline $\begin{array}{c}\mathrm{Y} 2 \\
9 \\
\end{array}$ & Entrega a Contratistas & $\begin{array}{c}\text { Mide el nivel en que el modelo es } \\
\text { desarrollado para integrarse con la fase } \\
\text { de construcción }\end{array}$ \\
\hline \begin{tabular}{c|}
$\mathrm{Y} 2$ \\
10 \\
\end{tabular} & 7D - FM / OM & $\begin{array}{c}\text { Mide el nivel en que el modelo es } \\
\text { desarrollado para apoyar la fase de } \\
\text { Operación y Mantenimiento. }\end{array}$ \\
\hline Y3 & $\begin{array}{l}\text { Nivel de madurez } \\
\text { BIM General }\end{array}$ & $\frac{Y 1+Y 2}{2}$ \\
\hline
\end{tabular}

\subsection{Variables dependientes}

Las variables dependientes fueron tomadas de la herramienta de Prosci que mide el Nivel de Madurez de la Gestión del Cambio en una organización o empresa. En este caso se utilizaron las 50 variables ordinales contenidas en el modelo, las cuales fueron agrupadas en 6 variables principales para llevar a cabo las correlaciones. Se utilizó una escala Likert de 1 a 5 :

Nivel 1: ausencia o nivel inicial

Nivel 2: presencia en proyectos aislados

Nivel 3: presencia en múltiples proyectos

Nivel 4: estandarización organizacional
Nivel 5: competencia organizacional

Las variables en cuestión son:

Tabla 2: Variables Independientes: Nivel de Madurez BIM. Fuente: elaboración propia a partir del Modelo de Auditoría de la Madurez de la Gestión del Cambio de Prosci, p67

\section{Variable X1: Liderazgo}

$\mathrm{X} 1_{1}$ : Patrocinio para Institucionalizar el cambio en la organización

$\mathrm{X}_{2}$ : Comunicación de los líderes claves sobre la importancia de gestionar la parte social del cambio

$\mathrm{X}_{3}$ : Fuerza de la coalición de formada para implementar el cambio

$\mathrm{X} 1_{4}$ : Visión para los esfuerzos de implementación del cambio

$\mathrm{X}_{1}$ : Fondos y recursos destinados para implementar el cambio

$\mathrm{X} 1_{6}$ : Accesibilidad de los líderes, toma de decisiones y compromiso con el equipo de proyecto para con el cambio.

$\mathrm{X} 1_{7}$ : Revisión por parte de los líderes de las prácticas de cambio en las evaluaciones de los proyectos

$\mathrm{X} 1_{8}$ : Creación de reglas, políticas y procedimientos que establecen los requerimientos del cambio y los refuerza

Variable X2:Aplicación

$\mathrm{X} 2_{1}$ : Porcentaje de proyectos donde se aplicó de forma estructurada el cambio

$\mathrm{X} 2_{2}$ : Metodologías y herramientas comunes y consistentes para aplicar el cambio

$\mathrm{X} 2_{3}$ : Funciones, divisiones o unidades que han aplicado el cambio $\mathrm{X} 2_{4}$ : Tipos de proyectos donde se aplicó el cambio

$\mathrm{X} 2_{5}$ : Disponibilidad de Herramientas para manejar el lado social del cambio

$\mathrm{X} 2_{6}$ : Recursos disponibles para la aplicación de los procesos y las herramientas de cambio en los proyectos

$\mathrm{X} 2_{7}$ : Presupuesto y fondos disponibles para la gestión del cambio en proyectos e iniciativas

$\mathrm{X} 2{ }_{8}$ : Aplicación de la gestión del cambio en los procesos y entregables

\section{Variable X3: Competencias}

$\mathrm{X} 3_{1}$ : Entrenamiento dado a los practicantes en el proceso de cambio

$\mathrm{X}_{2}$ : Habilidades demostradas de los practicantes poseen para llevar a cabo el cambio

$\mathrm{X}_{3}$ : Entrenamiento de los directivos y ejecutivos para efectuar el cambio y ejercer como patrocinadores del mismo

X34: Demostración de competencias de los directivos y los líderes ejecutivos para llevar a cabo el cambio

$\mathrm{X} 3_{5}$ : Entrenamiento de los administradores y supervisores para guiar la implementación del cambio

X36: Demostración de competencias de coaching de administradores y supervisores para guiar el cambio

$\mathrm{X} 3_{7}$ : Entrenamiento de los trabajadores para enfrentar el cambio

$\mathrm{X} 3_{8}$ : Capacidades demostradas por los trabajadores para llevar a cabo el cambio

$\mathrm{X} 3_{9}$ : Entrenamiento de los equipos de proyecto para enfrentar el cambio

\section{Variable X4: Estandarización}

$\mathrm{X} 4_{1}$ : Disponibilidad de estándares para implementar el cambio

$\mathrm{X} 4_{2}$ : Disponibilidad de un set de herramientas estandarizado para el cambio

$\mathrm{X}_{3}$ : Disponibilidad de un grupo o departamento encargado de la gestión del cambio

$\mathrm{X} 44$ : Roles y puestos necesarios para el cambio

$\mathrm{X}_{5}$ : Grupos de expertos en el tema de gestión del cambio

$\mathrm{X} 4_{6}$ : Redes para discutir y compartir sobre el cambio 


\begin{tabular}{c}
$\mathrm{X} 4_{7}$ : Currículo de competencias y se ha dado entrenamiento para \\
alcanzarlo \\
$\begin{array}{c}\mathrm{X} 4_{8} \text { : Integración del proceso de cambio con sistemas y herramientas } \\
\text { afines (Lean, Six Sigma, Planeación Estratégica, BPR. etc.) }\end{array}$ \\
$\begin{array}{c}\text { Variable } \mathbf{X} 5 \text { : Socialización } \\
\mathrm{X} 5_{1} \text { : Capítulo ejecutivo para construir las capacidades y competencias } \\
\text { necesarias }\end{array}$ \\
\hline $\mathrm{X} 5_{2}$ : Valor percibido de la gestión del cambio dentro de la organización \\
\hline $\mathrm{X} 5_{3}$ : Enganche y apoyo para el implementar el cambio \\
\hline $\mathrm{X} 5_{4}$ : Visión unificada del cambio a través de la empresa \\
\hline $\mathrm{X} 5_{6}$ : Comunicación del cambio implementado \\
\hline $\mathrm{X} 5_{7}$ : Difusión y publicación de los éxitos alcanzados en el cambio \\
\hline $\mathrm{X} 5_{8}:$ Fusión de la cultura de la empresa con el valor de gestionar el \\
cambio de forma efectiva \\
\hline Variable X6: Nivel de Madurez de la Gestión del Cambio \\
$X 1+X 2+X 3+X 4+X 5$ \\
5
\end{tabular}

\subsection{Correlación de variables}

Debido a la naturaleza ordinal de las variables definidas, así como el tamaño de la muestra de la población y la ausencia de supuestos dentro de la misma, se decidió optar por realizar una prueba no paramétrica de la estadística inferencial. La prueba seleccionada fue el Coeficiente de Correlación Rho de Spearman, mediante el cual se puede estimar la correlación o el grado de asociación entre dos variables, a partir del orden de los rangos de las puntuaciones de cada una de las variables que vamos a relacionar. Las correlaciones entre variables fueron conducidas de la siguiente manera.

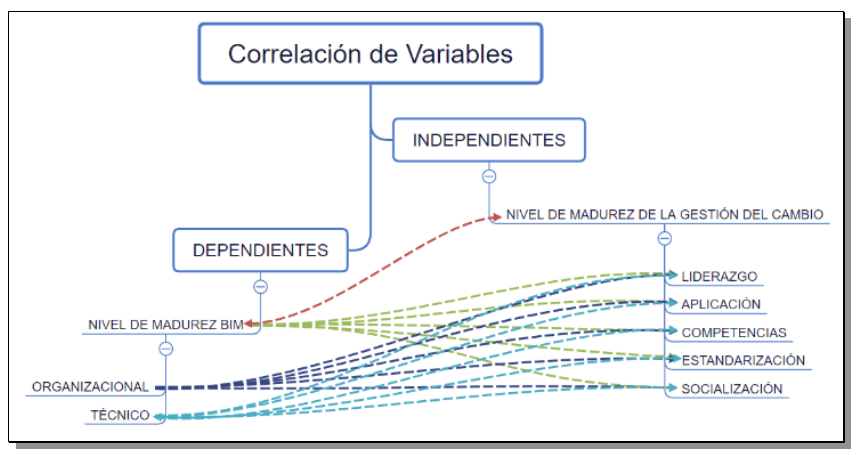

Figura 1: Correlación entre variables.

\section{Resultados y discusión}

\subsection{Análisis descriptivo}

En los resultados obtenidos se puede observar que en los resultados la totalidad de las empresas clasifican como PYMES, al menos en cuanto al número de empleados se refiere. De ellas el 50\% son consideradas Pequeñas Empresas. Este resultado está en concordancia con las estadísticas ofrecidas por el INEGI donde se señala que en el año 2015 el $97.6 \%$ de todas las empresas del país son PYMES.

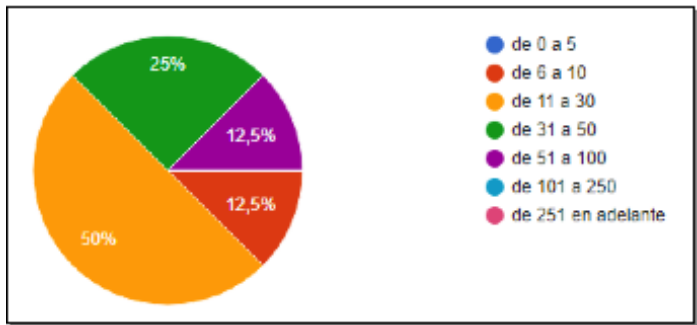

Figura 2: Número de empleados.

Desde el punto de vista de la actividad económica el mayor porcentaje ocupan las empresas dedicadas a los Servicios de Arquitectura Virtual con un $37.5 \%$. Tanto la Gerencia de Proyectos como la Edificación de Inmuebles comerciales ostentan un $25 \%$. La Supervisión de Obras se queda con un $12 \%$. Esto es coherente con la literatura estudiada donde en otras investigaciones exploratorias se enfatiza que BIM es utilizado por los despachos de diseño en mayor medida.

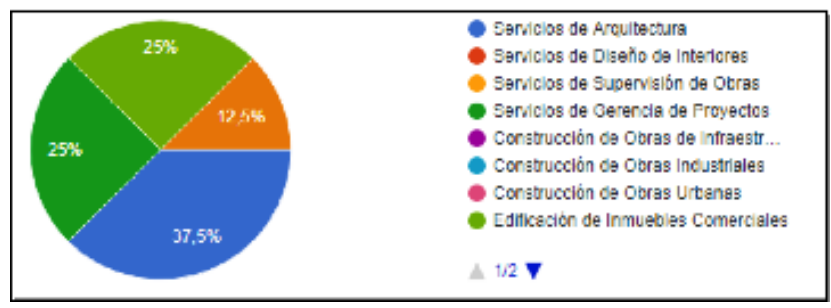

Figura 3: Actividad Económica

En cuanto a las fechas de inicio del proceso de Implementación BIM, se ha podido constatar que la empresa más longeva usando BIM comenzó en el 2007 y las más jóvenes en el 2014. Esto demuestra que se cumplió el objetivo de preseleccionar empresas con 3 o más años de experiencia en el uso de BIM. Por otra parte, en cuanto al costo que ha tenido el proceso de implementación BIM podemos constatar cómo el 50\% lo sitúan entre 100000 y 250000 pesos. Esta información concuerda con la aportada por consultoras BIM mexicanas, las cuales tasaron en entrevistas previas dicho rango como el valor promedio de los servicios de implementación y capacitación que ellos ofrecen.

Figura 4: Costos de la Implementación BIM.

Adentrándonos en los valores aportados por las variables independientes y dependientes podemos observar como el Nivel de Madurez promedio para todas las variables sobrepasa los 3.6 puntos (ver Ilustración 6). Esto nos lleva a considerar que de forma general la muestra estudiada está por encima del umbral mínimo de 3 establecido para nuestra

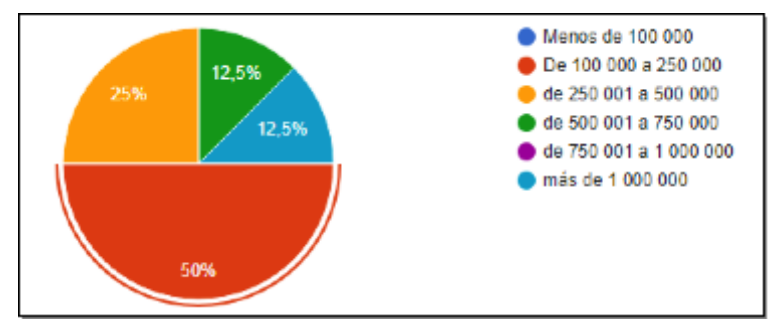

investigación. De forma más específica podemos ver como las variables globales como el Nivel de Madurez BIM y el 
Nivel de Madurez de la Gestión del Cambio, tienen valores muy cercanos, 3.8766 y 3.7905 respectivamente.

Tabla 3: Variables de la herramienta BIM-MM (variables dependientes de la investigación)

\begin{tabular}{|l|r|r|r|r|r|r|}
\hline & \multicolumn{1}{|c|}{$\mathrm{N}$} & \multicolumn{1}{|c|}{ Mínimo } & \multicolumn{1}{c|}{ Máximo } & \multicolumn{1}{l|}{ Media } & Desv. típ. & Varianza \\
\hline LIDERAZGO EN GC & 7 & 2.89 & 4.33 & 3.7778 & .49275 & .243 \\
APLICACIÓN EN GC & 7 & 2.78 & 4.67 & 3.9365 & .65060 & .423 \\
COMPETENCIAS EN GC & 7 & 2.67 & 4.50 & 3.7024 & .64344 & .414 \\
ESTANDARIZACIÓN EN & 7 & 3.00 & 4.22 & 3.8571 & .44312 & .196 \\
GC & 7 & 2.88 & 4.88 & 3.6786 & .64895 & .421 \\
SOCIALIZACIÓN EN GC & 7 & & & & \\
NIVEL DE MADUREZ DE & 7 & 2.84 & 4.41 & 3.7905 & .52368 & .274 \\
LA GESTIÓN DEL & & & & & & \\
CAMBIO & 7 & 3.18 & 4.45 & 4.0390 & .48349 & .234 \\
MADUREZ BIM & 7 & 2.73 & 4.36 & 3.7143 & .58312 & .340 \\
ORGANIZACIONAL & 7 & 2.95 & 4.41 & 3.8766 & .50936 & .259 \\
MADUREZ BIM TÉCNICA & 7 & & & & & \\
MADUREZ BIM & & & & & \\
N válido (según lista) & & 7 &
\end{tabular}

\subsection{Análisis estadístico}

El nivel de madurez de BIM vs las 5 variables de madurez. de la gestión del cambio

El primer análisis de correlación se establece entre cada una de las variables de la Gestión del Cambio y el Nivel de Madurez BIM global de las empresas encuestadas. Los resultados indican que existe una correlación positiva en todos los casos analizados, sin embargo, las correlaciones y significativas para un 0.05 están determinadas por las siguientes variables:

- $\quad$ Liderazgo $(r=0.88 \mathrm{~s}=0.008)$

- Competencias $(r=0.78 \mathrm{~s}=0.0036)$

Tablas 4: Variables de la herramienta BIM-MM (variables dependientes de la investigación)

\begin{tabular}{|c|c|c|c|c|}
\hline & & & $\begin{array}{l}\text { MADUREZ } \\
\text { BIM }\end{array}$ & $\begin{array}{l}\text { LIDERAZGO } \\
\text { EN GC }\end{array}$ \\
\hline \multirow[t]{2}{*}{ Rho de Spearman } & MADUREZ BIM & $\begin{array}{l}\text { Coeficiente de correlación } \\
\text { Sig. (bilateral) } \\
\text { N }\end{array}$ & 1.000 & $\begin{array}{r}.883^{\prime \prime} \\
.008 \\
7\end{array}$ \\
\hline & LIDERAZGO EN GC & $\begin{array}{l}\text { Coeficiente de correlación } \\
\text { Sig. (bilateral) } \\
\text { N }\end{array}$ & $\begin{array}{r}.883^{m+} \\
.008 \\
7\end{array}$ & 1.000 \\
\hline
\end{tabular}

. La correlación es significativa al nivel 0,01 (bilateral).

Tabla 5 - Correlación Madurez BIM vs Aplicación en GC

\begin{tabular}{|c|c|c|c|c|}
\hline & & & $\begin{array}{c}\text { MADUREZ } \\
\text { BIM }\end{array}$ & $\begin{array}{c}\text { APLICACIÓN } \\
\text { EN GC }\end{array}$ \\
\hline \multirow[t]{2}{*}{ Rho de Spearman } & MADUREZ BIM & $\begin{array}{l}\text { Coeficiente de correlación } \\
\text { Sig. (bilateral) }\end{array}$ & 1.000 & $\begin{array}{l}.631 \\
.129 \\
7\end{array}$ \\
\hline & APLICACIÓN EN GC & $\begin{array}{l}\text { Coeficiente de correlación } \\
\text { Sig. (bilateral) }\end{array}$ & $\begin{array}{r}.631 \\
.129 \\
7\end{array}$ & 1.000 \\
\hline
\end{tabular}

Tabla 6 - Correlación Madurez BIM vs Aplicación en GC

\begin{tabular}{|c|c|c|c|c|}
\hline & & & $\begin{array}{c}\text { MADUREZ } \\
\text { BIM }\end{array}$ & $\begin{array}{l}\text { COMPETENN } \\
\text { CIAS EN GC }\end{array}$ \\
\hline \multirow[t]{2}{*}{ Rho de Spearman } & MADUREZ BIM & $\begin{array}{l}\text { Coeficiente de correlación } \\
\text { Sig. (bilateral) } \\
\text { N }\end{array}$ & 1.000 & $\begin{array}{r}.786^{\circ} \\
.036 \\
7\end{array}$ \\
\hline & COMPETENNCIAS EN GC & $\begin{array}{l}\text { Coeficiente de correlación } \\
\text { Sig. (bilateral) } \\
\mathrm{N}\end{array}$ & $\begin{array}{r}.786 \\
.036 \\
7\end{array}$ & 1.000 \\
\hline
\end{tabular}

Tabla 7 - Correlación Madurez BIM vs Estandarización en GC

\begin{tabular}{|c|c|c|c|c|}
\hline & & & $\begin{array}{c}\text { MADUREZ } \\
\text { BIM }\end{array}$ & $\begin{array}{l}\text { ESTANDARIZ } \\
\text { ACIÓN EN GC }\end{array}$ \\
\hline \multirow[t]{2}{*}{ Rho de Spearman } & MADUREZ BIM & $\begin{array}{l}\text { Coeficiente de correlación } \\
\text { Sig. (bilateral) } \\
N\end{array}$ & 1.000 & $\begin{array}{r}.371 \\
.413 \\
7\end{array}$ \\
\hline & ESTANDARIZACIÓN EN GC & $\begin{array}{l}\text { Coeficiente de correlación } \\
\text { Sig. (bilateral) } \\
\text { N }\end{array}$ & $\begin{array}{r}.371 \\
.413 \\
7\end{array}$ & 1.000 \\
\hline
\end{tabular}

Tabla 8 - Correlación Madurez BIM vs Socialización en GC

\begin{tabular}{|c|c|c|c|c|}
\hline & & & $\begin{array}{c}\text { MADUREZ } \\
\text { BIM }\end{array}$ & $\begin{array}{c}\text { SOCIALIZACI } \\
\text { ON EN GC }\end{array}$ \\
\hline \multirow[t]{2}{*}{ Rho de Spearman } & MADUREZ BIM & $\begin{array}{l}\text { Coeficiente de correlación } \\
\text { Sig. (bilateral) } \\
N\end{array}$ & $\begin{array}{r}1.000 \\
7\end{array}$ & $\begin{array}{r}.414 \\
.355 \\
7\end{array}$ \\
\hline & SOCIALIZACIÓN EN GC & $\begin{array}{l}\text { Coeficiente de correlación } \\
\text { Sig. (bilateral) } \\
N\end{array}$ & $\begin{array}{r}.414 \\
.355 \\
7\end{array}$ & 1.000 \\
\hline
\end{tabular}

El nivel de madurez de BIM en el proyecto organizacional vs las 5 variables de madurez de la gestión del cambio

En este caso la correlación se establece entre el Nivel de Madurez BIM en el Proyecto (Organizacional) y las 5 variables del Nivel de Madurez de la Gestión del Cambio. En este caso solo el Liderazgo tiene una correlación positiva y significativa.

Tabla 9-12: Correlación Madurez BIM Técnica vs Socialización en GC.

\begin{tabular}{|c|c|c|c|c|}
\hline & & & $\begin{array}{l}\text { MADUREZ } \\
\text { BIM ENEL EL } \\
\text { PROYECYY } \\
\end{array}$ & $\begin{array}{c}\text { LIDERAZGO } \\
\text { EN GC }\end{array}$ \\
\hline \multirow[t]{2}{*}{ Rho de Spearman } & MADUREZ BIM EN EL PROYECYO & $\begin{array}{l}\text { Coeficiente de correlación } \\
\text { Sig. (bilateral) } \\
\text { N }\end{array}$ & 1.000 & $\begin{array}{r}.785^{\circ} \\
.036 \\
7 \\
\end{array}$ \\
\hline & LIDERAZGO EN GC & $\begin{array}{l}\text { Coeficiente de correlación } \\
\text { Sig. (bilateral) } \\
\text { N }\end{array}$ & $\begin{array}{r}.785^{\circ} \\
.036 \\
7 \\
\end{array}$ & 1.000 \\
\hline
\end{tabular}

El nivel de madurez de BIM tecnica vs las 5 variables de madurez de la gestión del cambio

En este caso la correlación se establece entre el Nivel de Madurez BIM Técnico y las 5 variables del Nivel de Madurez de la Gestión del Cambio. En este caso solo la variable Competencias tiene una correlación positiva y significativa.

Tabla 10 - Correlación Madurez BIM en el Proyecto vs Competencias en GC

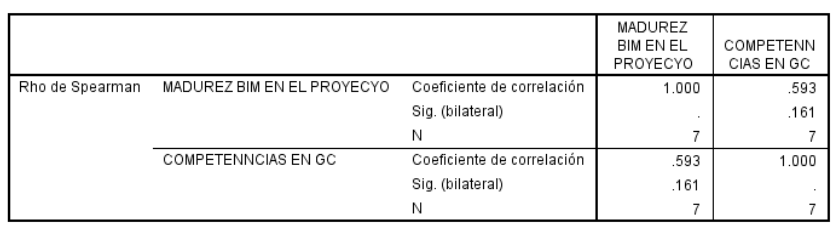

Tabla 11 - Correlación Madurez BIM en el Proyecto vs Estandarización en GC

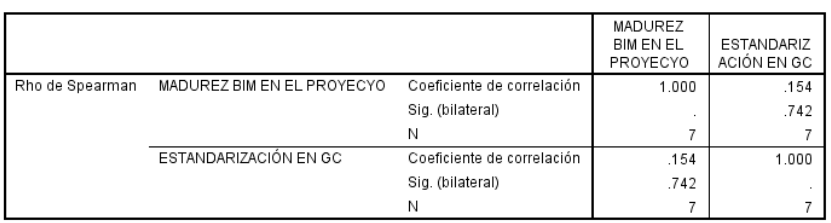


Tabla 12 - Correlación Madurez BIM en el Proyecto vs Socialización en GC

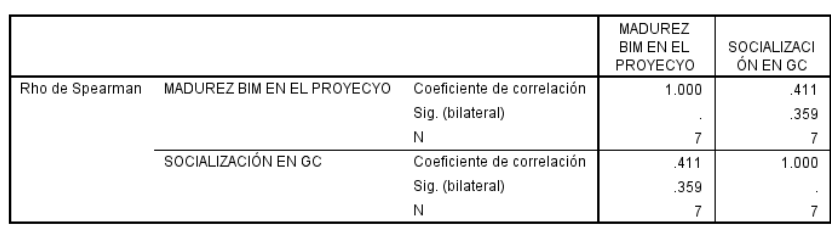

En este caso la correlación se establece entre el Nivel de Madurez BIM en el Proyecto (Organizacional) y las 5 variables del Nivel de Madurez de la Gestión del Cambio. En este caso solo el Liderazgo tiene una correlación positiva y significativa.

3.2.3 El Nivel de Madurez BIM Técnica vs Las 5 variables de Madurez de la Gestión del Cambio

Tabla 13 - Correlación Madurez BIM Técnica vs Aplicación en GC

\begin{tabular}{|lll|r|r|}
\hline & & & \multicolumn{1}{|c|}{ MADUREZ } & APLICACIÓN \\
BIM TÉCNICA & EN GC \\
\hline Rho de Spearman & MADUREZ BIM TÉCNICA & Coeficiente de correlación & 1.000 & .609 \\
& & Sig. (bilateral) & .147 \\
& & $\mathrm{~N}$ & 7 \\
\cline { 2 - 5 } & & Coeficiente de correlación & .609 & 1.000 \\
& APLICACIÓN EN GC & .147 & \\
& & Sig. (bilateral) & 7 & 7 \\
\hline
\end{tabular}

Tabla 14 - Correlación Madurez BIM Técnica vs Socialización en GC

\begin{tabular}{|c|c|c|c|c|}
\hline & & & $\begin{array}{c}\text { MADUREZ } \\
\text { BIM TÉCNICA }\end{array}$ & $\begin{array}{l}\text { COMPETENN } \\
\text { CIAS EN GC }\end{array}$ \\
\hline \multirow[t]{2}{*}{ Rho de Spearman } & MADUREZ BIM TÉCNICA & $\begin{array}{l}\text { Coeficiente de correlación } \\
\text { Sig. (bilateral) } \\
\text { N }\end{array}$ & 1.000 & $\begin{array}{r}.775^{\circ} \\
.041 \\
7\end{array}$ \\
\hline & COMPETENNCIAS EN GC & $\begin{array}{l}\text { Coeficiente de correlación } \\
\text { Sig. (bilateral) } \\
\mathrm{N}\end{array}$ & $\begin{array}{r}.775^{\circ} \\
.041 \\
7\end{array}$ & 1.000 \\
\hline
\end{tabular}

Tabla 15 - Correlación Madurez BIM Técnica vs Estandarización en GC

\begin{tabular}{|lll|r|r|}
\hline & & & MADUREZ & $\begin{array}{r}\text { ESTANDARIZ } \\
\text { ACIÓN EN GC }\end{array}$ \\
\hline BIM TÉCNICA de Spearman & MADUREZ BIM TÉCNICA & Coeficiente de correlación & 1.000 & .393 \\
& & Sig. (bilateral) & .384 \\
& & $\mathrm{~N}$ & 7 \\
\cline { 2 - 5 } & & .393 & 1.000 \\
& ESTANDARIZACIÓN EN GC & Coeficiente de correlación & .384 & \\
& & Sig. (bilateral) & 7 & 7 \\
\cline { 2 - 5 } & $\mathrm{N}$ & &
\end{tabular}

Tabla 16 - Correlación Madurez BIM Técnica vs Socialización en GC

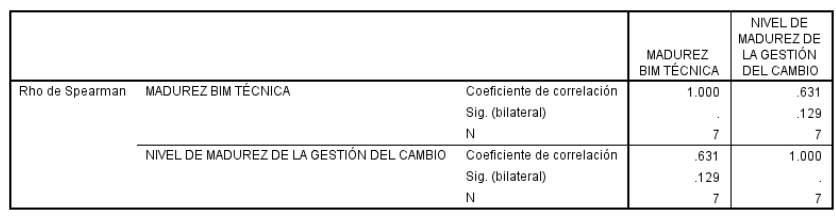

En este caso la correlación se establece entre el Nivel de Madurez BIM Técnico y las 5 variables del Nivel de Madurez de la Gestión del Cambio. En este caso solo la variable Competencias tiene una correlación positiva y significativa.

3.2.4 El Nivel de Madurez BIM vs El Nivel de Madurez de la Gestión del Cambio
Tabla 17 - Correlación Madurez BIM Técnica vs Socialización en GC

\begin{tabular}{|c|c|c|c|}
\hline & & $\begin{array}{c}\text { MADUREZ } \\
\text { BIM }\end{array}$ & $\begin{array}{l}\text { NIVEL DE } \\
\text { MADUREZ DE } \\
\text { LA GESTIÓN } \\
\text { DEL CAMBIO } \\
\end{array}$ \\
\hline MADUREZ BIM & $\begin{array}{l}\text { Correlación de Pearson } \\
\text { Sig. (bilateral) } \\
\mathrm{N}\end{array}$ & $\begin{array}{l}1 \\
7\end{array}$ & $\begin{array}{r}.834 \\
.020 \\
7\end{array}$ \\
\hline $\begin{array}{l}\text { NIVEL DE MADUREZ DE } \\
\text { LA GESTIÓN DEL } \\
\text { CAMBIO }\end{array}$ & $\begin{array}{l}\text { Correlación de Pearson } \\
\text { Sig. (bilateral) } \\
\text { N }\end{array}$ & $\begin{array}{r}.834^{x} \\
.020 \\
7\end{array}$ & $\begin{array}{l}1 \\
7\end{array}$ \\
\hline
\end{tabular}

*. La correlación es significante al nivel 0,05 (bilateral).

\section{Conclusiones}

Partiendo de los objetivos planteados, hemos podido constatar que BIM ha ido trascendiendo el etiquetado de Tecnología para convertirse en una filosofía que, a través de una metodología más vasta, influye en aspectos más amplios que incluyen, pero no se limitan a: los procesos, las tecnologías, los comportamientos y las políticas de una organización u empresa. Por otra parte la exploración de la Gestión del Cambio como alternativa resulta ser una variante que tiene puntos de contacto importantes con los modelos BIM estudiados, sobre todo a nivel organizacional e individual, dentro del diseño y construcción sostenible.

En otro orden hemos identificado Modelos de Madurez tanto para BIM como para la Gestión del Cambio, que consideramos como herramientas adecuadas para establecer mediciones y comparaciones entre las distintas capacidades que puedan tener las empresas. En la conformación y aplicación del cuestionario llegamos a la conclusión de que a pesar de que es posible medir las variables trabajadas, lo ideal sería hacerlo de forma presencial para tener mayor certeza de los resultados y no estar expuestos a un criterio de interpretación de los usuarios.

Debemos rescatar además que, los resultados de las mediciones presentan un balance positivo en torno a nuestra hipótesis. En primera instancia la correlación entre el Nivel de Madurez BIM y el Nivel de Madurez de la Gestión del Cambio, existe de forma fuerte y significativa. Hemos concluido que esta correlación está dada por las variables Liderazgo y Competencias, dentro del diseño y construcción sostenible. Este hecho que está acorde con otros estudios similares realizados por Prosci y publicado en sus en sus informes anuales.

Hay que recalcar el carácter parcial de estos resultados debido a que muchas otras variables pueden influir en esta correlación y no han sido tenidas en cuenta para la misma. En este sentido sería interesante que una investigación posterior pudiera destinarse al estudio e identificación de otras variables para dar paso a la conformación de un índice que caracterice de forma más completa el proceso de implementación de BIM en una empresa mexicana.

Finalmente consideramos que nuestra investigación abre una puerta a BIM desde una perspectiva integradora que incluye a la tecnología junto a procesos socio culturales inherentes al cambio, esto desde la perspectiva del diseño y construcción sostenible. Es en la conducción del cambio mismo donde somos de la opinión que deben centrarse las empresas y organizaciones que deseen alcanzar los mayores beneficios de BIM. Con nuestro trabajo ofrecemos la Gestión del Cambio como una alternativa viable para enfrentar un 
proceso de implementación de BIM que es ciertamente disruptivo e innovador.

English Summary

The influence of change management on the implementation of BIM in the sustainable building industry in Mexico.

\begin{abstract}
BIM has put the industry's traditional production mode in crisis to focus on collaborative and information management processes as fundamental pillars of a new paradigm. Despite its acknowledged benefits, the implementation and dissemination of BIM have numerous barriers that transcend the technological dimension to reach the social one. The latter is expressed in the change of work methodologies, sustainable design and building construction, in new forms of collaboration, in the appearance of new roles, in the adjustments of business organization, contracts and business models. All these aspects also have a common factor that is change, an element where the BIM implementation process finds its greatest obstacle due to the innovation it represents for any organization. In our research, a statistical correlation is carried out to determine the influence of Change Management in the BIM implementation process.
\end{abstract}

Keywords:

BIM, CM, Building Information Modeling, Change Management.

\section{Agradecimientos}

Al Programa de Maestría y Doctorado en Arquitectura de la UNAM por brindarme la oportunidad, al Padrón de CONACYT por hacer viable este tipo de intercambios y propuestas, a todos los profesores que de una forma u otra han contribuido a mi formación aportando sus valiosos conocimientos, en especial al director de tesis y comité tutor, a mi familia por estar siempre a mi lado, a mis amigos y todos aquellos que me brindaron su apoyo desinteresado.

\section{Referencias}

Calderisi, S. (2013). Building Information Modelling (BIM) Maturity in the UK Power Transmission Industry: Current Level and Common Factors Dedication.

Doppler, K., \& Lauterburg, C. (1998). Change Management. Managing Defence in a Democracy (1st ed.). Barcelona: Editorial Ariel, S. A.

Eastman, C., Teicholz, P., Sacks, R., \& Liston, K. (2011). BIM Handbook: A Guide to Building Information Modeling for Owners, Managers, Designers, Engineers and Contractors. Building (Vol. 2). https://doi.org/10.1002/9780470261309

Giel, B., Graduate Research Assistant, P. D., A Issa, R. R., Professor, H., Liu, R., \& Professor, A. (2012). PERCEPTIONS OF ORGANIZATIONAL BIM MATURITY VARIABLES WITHIN

THE US AECO INDUSTRY. In CIB W078 2012: 29th International Conference - Beirut, Lebanon, 17-19 October.

Hardin, B., \& McCool, D. (2014). BIM and Construction Management Proven tools, methods, and workflows. Igarss 2014 (second edi).

Prosci. (2017). ADKAR Model Overview. Retrieved from https://www.prosci.com/adkar/adkar- model

Randy Deutsch, AIA, L. A. (2011). BIM and Integrated Design: Strategies For Architectural Practice.

Zhurnal Eksperimental'noi i Teoreticheskoi Fiziki (1st ed.). John Wiley \& Sons, Inc.

Sadatsafavi, H., \& Walweski, J. (2011). Study of Organizational Change Management in Project- Driven Organizations Using Maturity Models. In PMI® Global Congress 2011-North America, Dallas, TX. Newtown Square (pp. 1-9). Project Management Institute.

Sebastian, R., \& van Berlo, L. (2010). Tool for Benchmarking BIM Performance of Design, Engineering and Construction Firms in The Netherlands. Architectural Engineering and Design Management. https://doi.org/10.3763/aedm.2010.IDDS3

Succar, B. (2009). Building information modelling framework: A research and delivery foundation for industry stakeholders. Automation in Construction, 18(3), 357-375. https://doi.org/10.1016/j.autcon.2008.10.003

Succar, B., \& Kassem, M. (2016). Building Information Modelling : Point of Adoption. CIB World Congres, (2016), 1-11.

Torres Aranda, L. A. (2012). Herramientas de Apoyo a la Gestión del Cambio organizacional en un Sistema de Lean Manufacturing, 100.

Wu, C., Xu, B., Mao, C., \& Li, X. (2017). OVERVIEW OF BIM MATURITY MEASUREMENT

TOOLS. Journal of Information Technology in Construction (ITcon), 22(22), 34-62. Retrieved from http://www.itcon.org/2017 